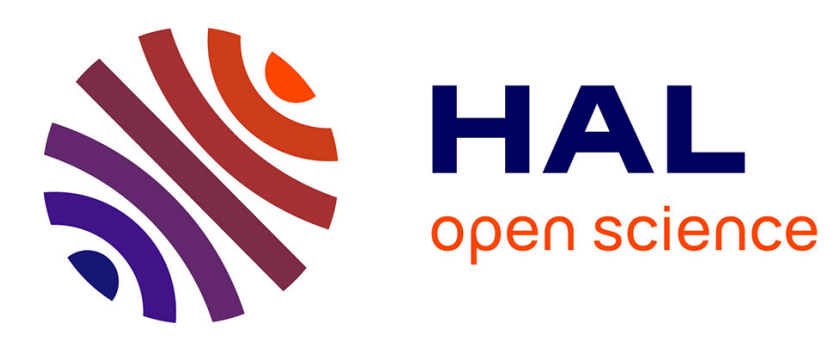

\title{
Transient Solution Determination of the Boltzmann Equation Using a Polynomial Expansion
}

\author{
S. Tabikh, C. Gontrand, J.-P. Nougier
}

\section{To cite this version:}

S. Tabikh, C. Gontrand, J.-P. Nougier. Transient Solution Determination of the Boltzmann Equation Using a Polynomial Expansion. Journal de Physique III, 1997, 7 (2), pp.281-289. 10.1051/jp3:1997121 . jpa-00249577

\section{HAL Id: jpa-00249577 https://hal.science/jpa-00249577}

Submitted on 1 Jan 1997

HAL is a multi-disciplinary open access archive for the deposit and dissemination of scientific research documents, whether they are published or not. The documents may come from teaching and research institutions in France or abroad, or from public or private research centers.
L'archive ouverte pluridisciplinaire HAL, est destinée au dépôt et à la diffusion de documents scientifiques de niveau recherche, publiés ou non, émanant des établissements d'enseignement et de recherche français ou étrangers, des laboratoires publics ou privés. 


\title{
Transient Solution Determination of the Boltzmann Equation Using a Polynomial Expansion
}

\author{
S. Tabikh $\left({ }^{1,2, *}\right)$, C. Gontrand $\left({ }^{2,3}\right)$ and J.P. Nougier $\left({ }^{4}\right)$ \\ (1) Université des Antilles et de la Guyane, boulevard Légitimus, BP 771 , \\ 97173 Pointe-à-Pitre Cedex, France \\ $\left({ }^{2}\right)$ Centre Inter-universitaire de Microélectronique de la Région Lyonnaise, \\ 20 avenue A. Einstein, 69621 Villeurbanne Cedex, France \\ $\left({ }^{3}\right)$ Laboratoire de Physique de La Matiere $\left(^{*}\right)$, Institut National des Sciences Appliquées de \\ Lyon, 20 avenue A. Einstein, 69621 Villeurbanne Cedex, France \\ ${ }^{(4)}$ Centre d'Électronique de Montpellier $\left({ }^{* *}\right)$. Université Montpellier II, \\ 34095 Montpellier Cedex 5, France
}

(Received 8 March 1996, revised 4 July and 20 September 1996, accepted 8 November 1996)

PACS.72.10.-d - Theory of electronic transport, scattering mechanisms PACS.63.20-e - Phonons in crystal lattices

\begin{abstract}
The transient and stationary Boltzmann equation are solved by using a LegendreLaguerre polynomial expansion for $\mathrm{p}-\mathrm{Si}$. Its resolution technique is based on a least-square fitting algorithm. We show this method to be as accurate as the exact ones (particular or direct iterative process); the great interest lies on its fast computation, roughly 200 times faster than a Monte-Carlo code for instance, for the same accuracy.
\end{abstract}

\section{Introduction}

The most effective ways to get the transient state of the Boltzmann equation (B.E.) are the iterative [1,2] and the Monte-Carlo (M.C.) [3] methods. The former one is well known to find directly, with a high level of accuracy, the distribution function itself. Then, the moments of the first and second order (mean velocity and energy, as well as diffusion coefficients), are derived in a straightforward manner. The latter one (M.C.) is another way to solve the B.E. It lays in a detailed microscopic description of a carrier ensemble using a random number hauling. But these algorithms are quite time consuming. On another hand, earlier works assumed the hypothesis that the distribution function could be developed along the two first terms of Legendre polynomials $[4,5]$. Indeed, the expansion of the distribution function, which depends on the magnitude and direction of the carrier wave vector, into spherical harmonics, leads to an infinite set of coupled differential equations which is very hard to handle. However, in certain limiting case the problem simplifies considerably. For instance, if the charges lose only a small fraction of their energy at each collision, the wave vector distribution is nearly

$\left({ }^{*}\right)$ Author for correspondence

(*) U.A. CNRS N 358

(*) URA CNRS No391 
spherically symmetric and only the first two Legendre polynomial are needed in its expansion, i.e.:

$$
f(k, \theta)=f_{0}(k)+f_{1}(k) \cos \theta
$$

$\theta$ is the angle the wave vector makes with the electric field.

This approximation is the well known diffusion one [6]. The physical intention behind this expansion is the separation of the function in a symmetric or diffusion term and a drift term weighted in the direction of field.

But, it has been shown that this method is no valid for high electric field, because of a large spreading of the distribution function along the field direction [7].

In the same manner, it has been proposed to assimilate the distribution function as a displaced Maxwellian [7,8]. This approximation is suitable for high electric field, but not far from the ohmic regime. For higher electric field, the distribution function presents a large non-symmetrical spread along the electric field direction. The shifted Maxwell-Boltzmann distribution function is somewhat doubtful because it requires that interaction between carriers are frequent enough to share energy and momentum between collision with other scatterers; model calculations by van Someren Greve [9] show that the shifted Maxwell-Boltzmann distribution function is a very poor approximation.

The distribution function has been also expanded using a set of displaced Maxwellian [10]. This method was no longer a good way to establish a "real base", for any electric field.

The aim of this paper is to develop a fast computation method, compared to usual ones, especially for high electric fields. Indeed, the key idea is also to develop the distribution using not only a generator system, but a actual base. This method, based on a Legendre and Laguerre polynomial expansion is described hereafter, in Section 2.

\section{Principle}

The carrier distribution function $f(\mathbf{k}, \mathbf{E}, t)$ is solution of the B.E. In the bulk, under a constant electric field and for transient regime, it leads to:

$$
\frac{\partial f(\mathbf{k}, \mathbf{E}, t)}{\partial t}=-\frac{e \mathbf{E}}{\hbar} \cdot \nabla_{k} f(\mathbf{k}, \mathbf{E}, t)+\left.\frac{\partial f}{\partial t}\right|_{\text {coll }} .
$$

The left term of the equation represents a change in the distribution due to particles being added or subtracted by scattering, by trapping or release of trapped particles, or by generation or recombination.

The first term on the right represents the acceleration driven by the electric field (E). The term $\left.\frac{\partial f}{\partial t}\right|_{\text {coll }}$ expresses the rapid changes due to scattering:

$$
\left.\frac{\partial f}{\partial t}\right|_{\text {coll }}=\iiint \mathrm{d}^{3} k^{\prime}\left[P\left(\mathbf{k}^{\prime}, \mathbf{k}\right) f\left(\mathbf{k}^{\prime}\right)-P\left(\mathbf{k}, \mathbf{k}^{\prime}\right) f(\mathbf{k})\right]
$$

$P\left(\mathbf{k}, \mathbf{k}^{\prime}\right)$ represents the rate at which a particle scatters from state $\mathbf{k}$ to $\mathbf{k}^{\prime}$.

The first term expresses the flow of particles into the state $\mathbf{k}$, the last term the loss by scattering into another state.

In direct iterative techniques [1], equation (1) is discretized in a straightforward manner. For example, in spherical coordinates with $E$ along the z-axis (see Fig. 1 ) $\mathbf{k}=\{k, \theta, \phi\}, \mathrm{f}(\mathbf{k}$ ) is determined at $k_{i}, \theta_{j}, \phi_{l}(0 \leq i \leq m-1,0 \leq j \leq n-1,0 \leq l \leq p-1)$.

In equation (1), E. $\nabla_{k} f=E \partial f(k) / \partial k$ can be expressed using central or forward, or backward differences, as a linear combination of $f_{i j l}=f\left(k_{i}, \theta_{j}, \phi_{1}\right)$. 


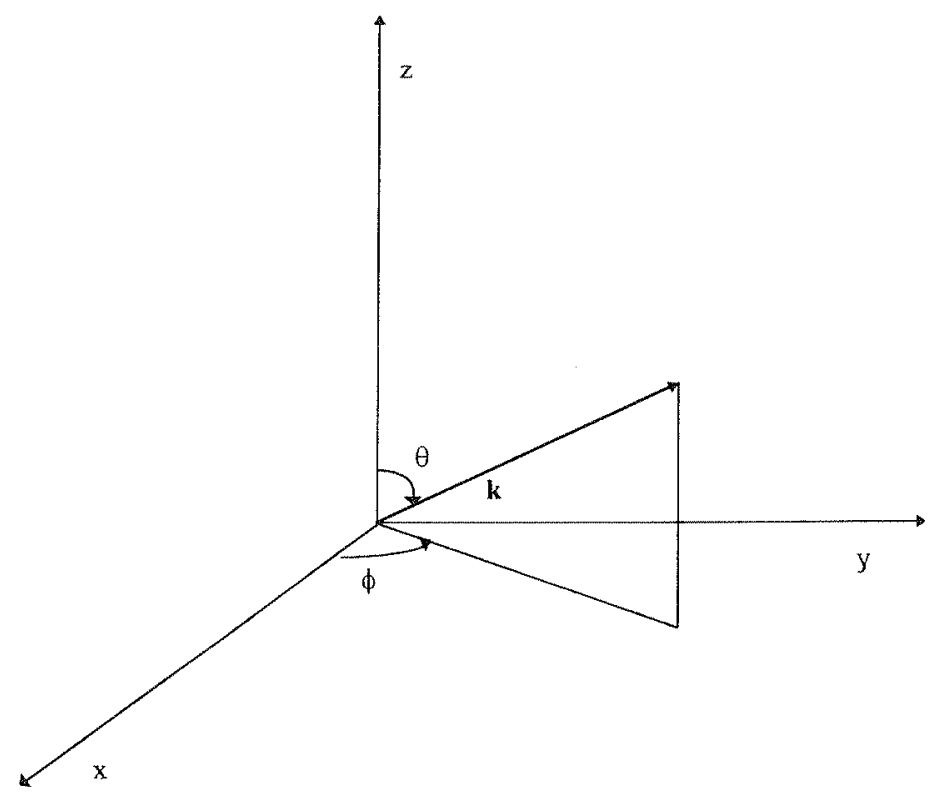

Fig. 1. Spherical coordinates.

In the same way, the integral of equation (1) using for example the Simpson rule, can be expressed as a linear combination of the $f_{i j l}$.

Finally the right side term of equation (1) can be written:

$$
\sum_{i, j, l} \mathrm{~A}_{i, j, l}^{i^{\prime}, j^{\prime}, l^{\prime}} f\left(k_{i}, \theta_{j}, \phi_{l}\right)
$$

where the coefficients $A_{i, j, l^{i^{\prime}}, l^{\prime}, l^{\prime}}=A\left(k_{i}, \theta_{j}, \phi_{l} ; k_{i}^{\prime}, \theta_{j}^{\prime}, \phi_{l}^{\prime}\right)$ do not depend on the unknowns $f_{i j l}$. They depend only on the electric field, on the semiconductor band shape, on the scattering mechanisms, on the methods used for the discretization procedure.

In this work, we consider that the conduction band is spherical; $f$ is symmetric around $\mathbf{E}$ and thus independent on $\phi$. However, it is supposed to be non degenerate and parabolic. On the other band, $P\left(\mathbf{k}, \mathbf{k}^{\prime}\right)$ deals with acoustic and optical scatterings.

Our method consists in expanding the transient distribution function using a set of fixed known functions. We write:

$$
f(\mathbf{k}, t)=f(k, \theta, t)=\sum_{s=0}^{+\infty} g_{s}(k, t) P_{s}(\cos \theta)
$$

where $P_{s}$ is the Legendre polynomial.

The expansion in a series of Legendre polynomials is usually referred to as a Legendre series. Its properties are quite similar to the familiar Fourier series. In particular, we can use the orthogonality property, to show that the series is unique.

We replace the wave vector $\mathbf{k}$ by the energy $\varepsilon$; then: $g_{s}(k, t)=\hat{g}_{s}(\varepsilon, t)$.

At thermal equilibrium, the carrier density is proportional to $\exp \left(-\varepsilon /\left(k_{\mathrm{B}} T\right)\right)$, that is:

$$
\hat{g}_{s}(\varepsilon, t)=\exp \left(\frac{-\varepsilon}{k_{\mathrm{B}} T}\right) h_{s}(\varepsilon, t) .
$$




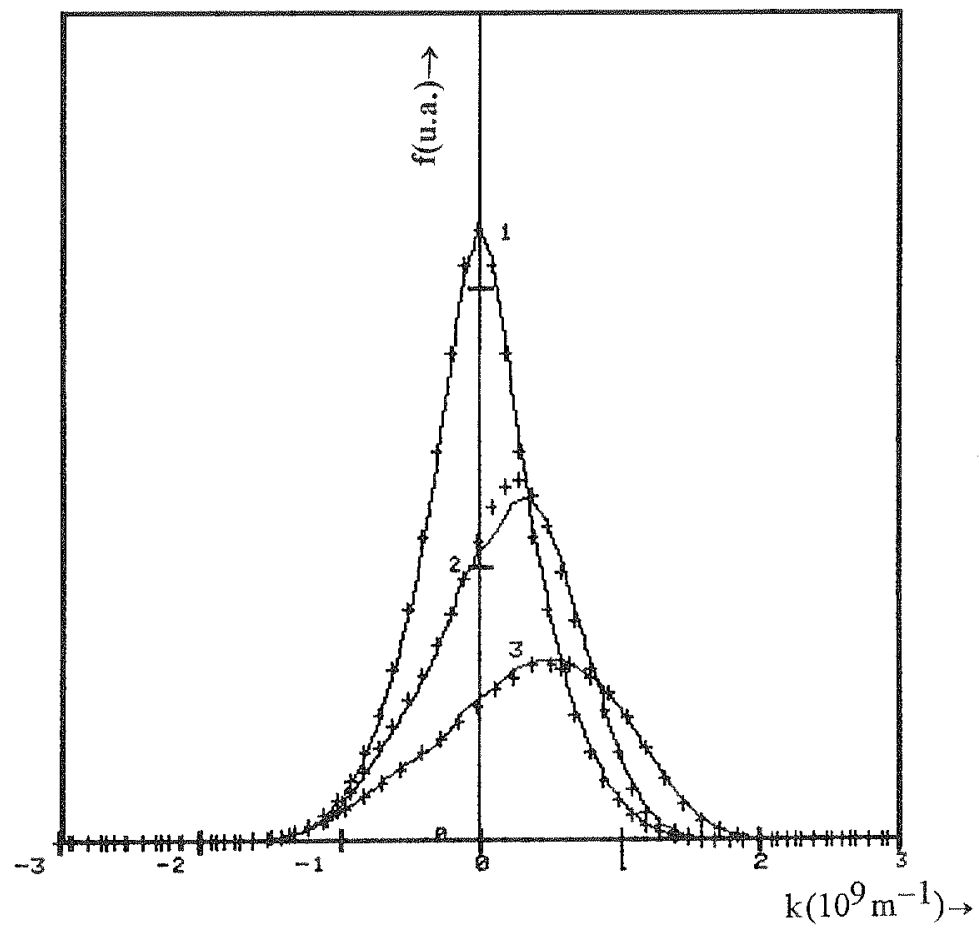

Fig. 2. Stationary distribution function, arbitrary unit (u.a.):(1):0 kV cm${ }^{-1}:(2): 5 \mathrm{kV} \mathrm{cm}^{-1}$; (3): $20 \mathrm{kV} \mathrm{cm}{ }^{-1}$; Legendre-Laguerre expansion: ( ) ; Monte-Carlo method: $(+++)$.

With the reduced variable, $x=\varepsilon /\left(k_{\mathrm{B}} T\right)$, we get:

$$
g_{s}(k, t)=\hat{g}_{s}(\varepsilon, t)=\exp \left(\frac{-\varepsilon}{k_{\mathrm{B}} T}\right) h_{s}(\varepsilon, t)=\exp (-x) \hat{h}_{s}(x, t)
$$

$h_{s}(x, t)$ is then expanded in an orthogonal Laguerre polynomial set:

$$
\hat{h}_{s}(x, t)=\sum_{m=0}^{+\infty} a_{s m}(t) L_{m}(x)
$$

$L_{m}(x)$ : Laguerre polynomial.

Considering a Legendre and Laguerre polynomial product allows us to split the $t$ (time) and $\mathrm{k}$ (wave vector) variables.

In practice, we have to calculate the $\mathbf{k}$ term only at the first beginuing of the computer program.

From (4) to (3), to(2), we get:

$$
f(\mathrm{k}, t)=\sum_{s=0}^{+\infty} \sum_{m=0}^{+\infty} a_{s m n}(t) \exp (-x) L_{m}(x) P_{s}(u)
$$

with $x=e /\left(k_{\mathrm{B}} T\right)$ and $u=\cos \theta$. 


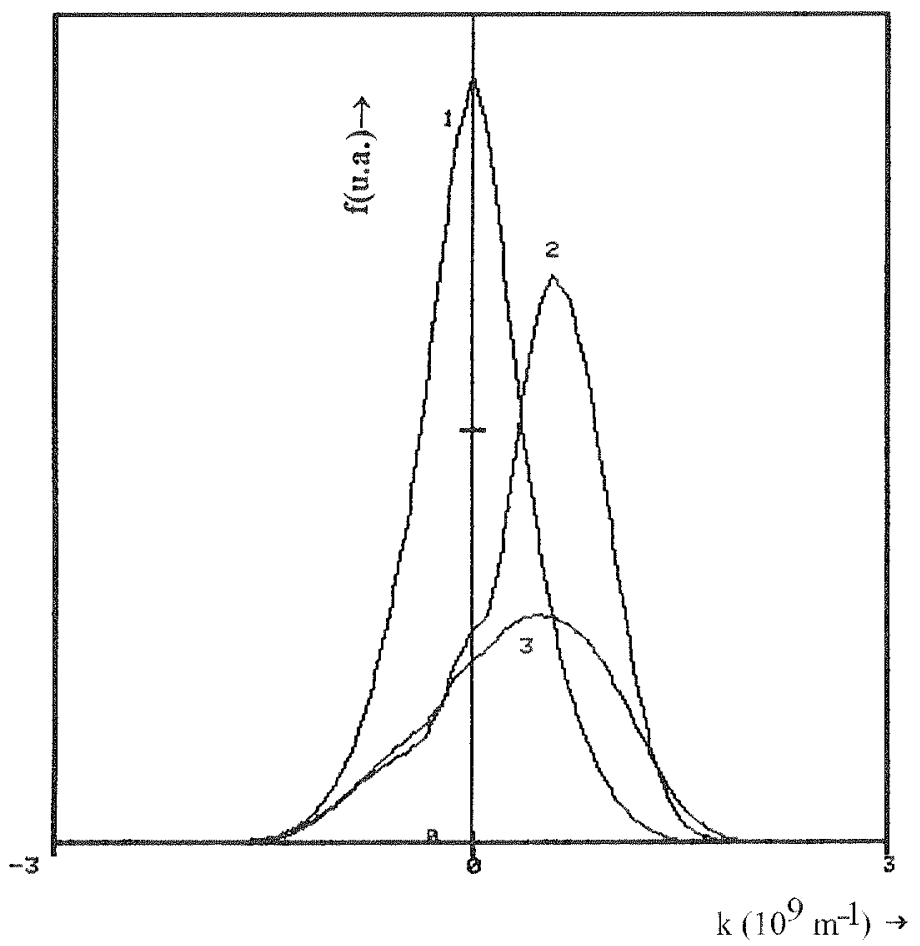

Fig. 3. - Transient distribution function, arbitrary unit (u.a.): $E=20 \mathrm{kV} \mathrm{cm}{ }^{-1} ;(1): t=0 \mathrm{ps}$; (2): $t=0.25 \mathrm{ps} ;(3): t=3.3 \mathrm{ps}$.

As well as $\cos \theta$, a Legendre polynomial is defined in the $[-1,+1]$ range. In the same way, Laguerre polynomial are defined on the $[0,+\infty[$, as well as the energy and therefore the wave vector module.

Finally, we use a finite function set, which gives:

$$
f(\mathbf{k}, t)=\sum_{s=0}^{I} \sum_{m=0}^{I} a_{s m}(t) \exp (-x) L_{m}(x) P_{s}(u)
$$

The principal benefit of treating equation (5) is that $t$ and $\mathbf{k}$ are now separated variables. As a key consequence, the new unknowns to deal with are then the $a_{s m}(t)$ ones.

The Legendre polynomials are defined in the $[-1,+1]$ interval by the recurrence relation:

$$
\left\{\begin{aligned}
& P_{0}(u)=1 \\
& P_{1}(u)=u \\
& P_{s+1}(u)=\frac{1}{s+1}\left[(2 s+1) u P_{s}(u)-s P_{b-1}(u)\right] \text { with } s \geq 1 .
\end{aligned}\right.
$$

Of course, we add the orthogonality relation of these polynomials:

$$
\int_{-1}^{+1} P_{s}(u) P_{i}(u) \mathrm{d} u=\frac{2}{2 s+1} \delta_{s i}
$$

where $\delta_{s i}$ is the Kronecker symbol. 


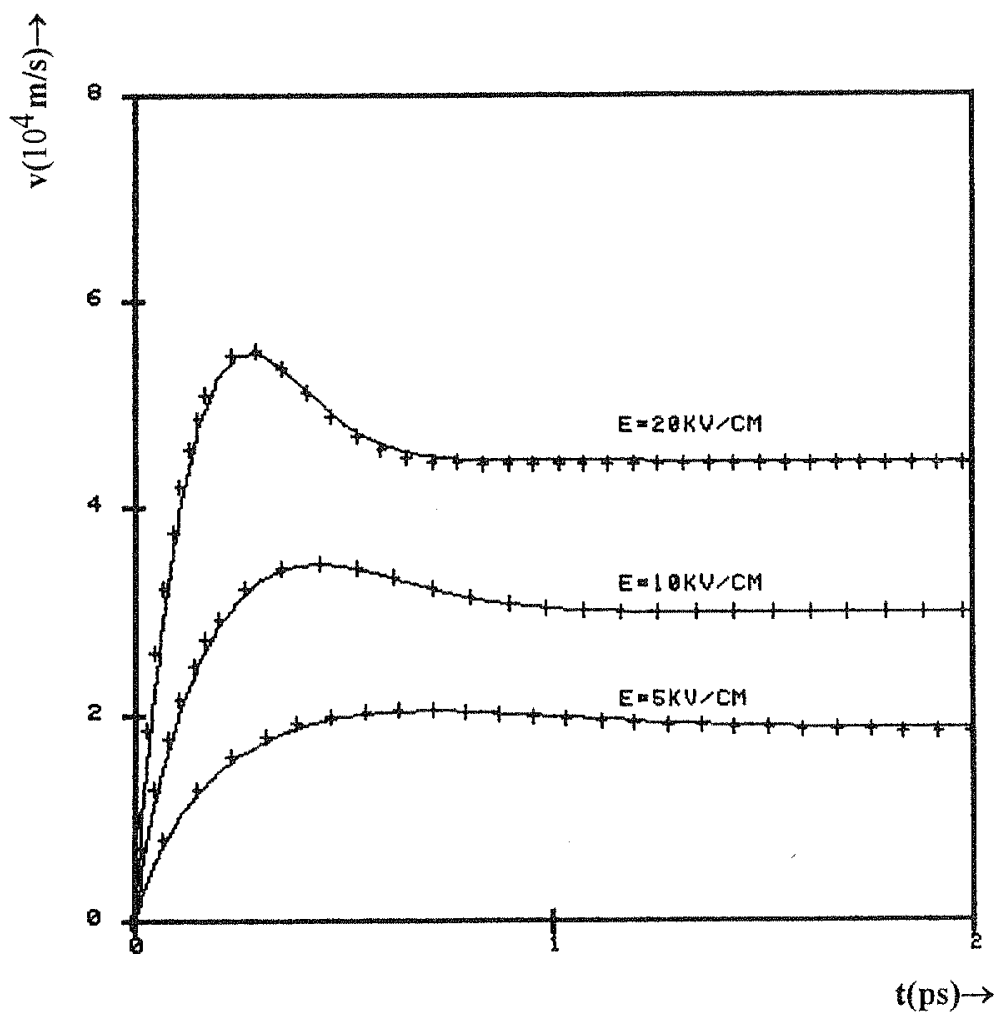

Fig. 4. - Carrier drift velocity; Legendre-Laguerre expansion: $(-)$; Monte-Carlo method: $(+++)$.

Concerning Laguerre polynomials, for the interval $[0,+\infty[$, they are given by:

$$
\left\{\begin{array}{c}
L_{0}(x)=1 \\
L_{1}(x)=-x+1 \\
L_{m+1}(x)=\frac{1}{m+1}\left[(2 m+1-x) L_{m}(x)-m L_{m-1}(x)\right] \quad \text { with } m \geq 1 .
\end{array}\right.
$$

The orthogonality relation of Laguerre polynomial is:

$$
\int_{0}^{+\infty} \exp (-x) L_{m}(x) L_{j}(x) \mathrm{d} x=\delta_{m j}
$$

\section{Technical Solving}

Substituting into the B.E. (1) $f(\mathbf{k}, \mathbf{E}, t)$ by its expression given by (5), we get:

$$
\sum_{s=0}^{I} \sum_{m=0}^{J} \exp (-x) L_{m}(x) P_{s}(u) \frac{\mathrm{d} a_{s m}(t)}{\mathrm{d} t}=\sum_{s=0}^{I} \sum_{m=0}^{J} a_{s m}(t) \Psi\left[\exp (-x) L_{m}(x) P_{s}(u)\right]
$$

with

$$
\Psi f(\mathbf{k}, \mathbf{E}, t)=-\frac{e \mathbf{E}}{\hbar} \cdot \nabla_{k} f(\mathbf{k}, \mathbf{E}, t)+\left.\frac{\partial f}{\partial t}\right|_{\text {coll }}
$$




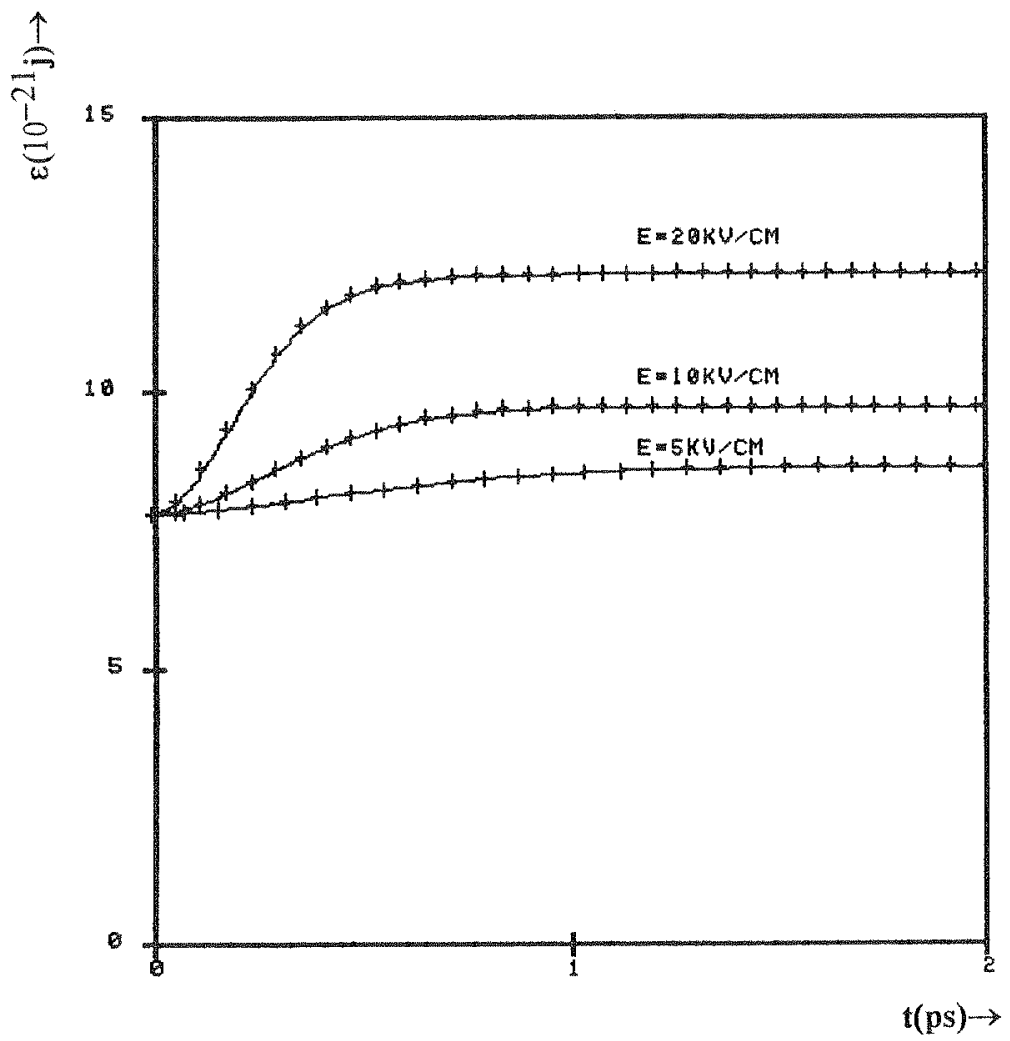

Fig. 5. - Carrier mean energy; Legendre-Laguerre expansion: (-) : VontemCarlo method: $(+++)$.

Multiplying equation $(10)$ by $P_{i}(u) L_{j}(x),(i=0,1, \ldots, I ; j=0,1, \ldots, J)$, and integrating over $u$ and $x$, we obtain:

$$
\sum_{s=0}^{I} \sum_{m=0}^{J} A_{i j}^{s m} \frac{\mathrm{d} a_{s m}(t)}{\mathrm{d} t}=\sum_{s=0}^{I} \sum_{m=0}^{J} B_{i j}^{s m} a_{s m}(t)
$$

with:

$$
\left\{\begin{array}{l}
A_{i j}^{s m}=\int_{0}^{+\infty} \exp (-x) L_{m}(x) L_{j}(x) \mathrm{d} x \int_{-1}^{+1} P_{s}(u) P_{i}(u) \mathrm{d} u \\
B_{i j}^{s m}=\int_{0}^{+\infty} L_{j}(x) \int_{-1}^{+1} P_{i}(u) \Psi\left[\exp (-x) L_{m}(x) P_{s}(u)\right] \mathrm{d} u \mathrm{~d} x
\end{array}\right.
$$

We may easily show, from orthogonality relations (7) and (9):

$$
A_{i j}^{s m}=\frac{2}{2 s+1} \delta_{s i} \delta_{m j}
$$

$B_{i j}^{s m}$ is time-independent, so that it can be calculated once for ever.

Introducing the value of $A_{i j}^{s m}$ into equation (11), we have:

$$
\frac{2}{2 i+1} \frac{\mathrm{d} a_{i j}(t)}{\mathrm{d} t}=\sum_{s=0}^{I} \sum_{m=0}^{J} B_{i j}^{s m} a_{s m}(t), \quad i=0,1, \ldots, I ; j=0,1, \ldots, J .
$$


Then, we get $n$ equations with $n$ unknowns set, with $n=(I+1)(J+1)$. It can be written in the following matrix form:

$$
\frac{\mathrm{d} \mathbf{a}}{\mathrm{d} t}=[H] \mathbf{a}(t)
$$

$\mathrm{a}(t)=\left\{a_{i j}(t)\right\}$ and $\frac{\mathrm{da}}{\mathrm{d} t}=\left\{\frac{\mathrm{d} a_{i j}}{\mathrm{~d} t}\right\}$ are column vectors (n rows, one column).

$$
[H]_{p, r}=H_{i j}^{s m} ; p=i(J+1)+j+1 ; r=s(J+1)+m+1 .
$$

Matrix $[H]$ is a $n \times n$ square matrix; for the same reason as the $B_{i j}^{s m}$, it will be first computed. Note that the $[\mathrm{H}]$ matrix is time independent.

Relation (12) represents a well known differential system; the solution is

$$
\mathbf{a}(t)=\exp ([H] t) \mathbf{a}(t=0),
$$

where $\mathbf{a}(t=0)$ refers to the initial function. We can then obtain so far a $(t)$, and consequently the distribution function at $t$ time.

We may also obtain the steady state; we have:

$$
\left(\frac{\mathrm{da}}{\mathrm{d} t}\right)_{t:=t_{s}}=0 .
$$

Considering (12), we may write:

$$
[H] \mathrm{a}\left(t=t_{s}\right)=0 .
$$

Because of truncation and discretization errors, the $[H]$ determinant is not exactly null. The method commonly employed is to give an arbitrary value to one of the unknown (in practice, we have chosen arbitrarily to set $a_{11}=1$ ). This way, system (13) becomes overdetermined (i.e., more unknowns than equations). We solve it by a least mean squares method. After calculating $\mathbf{a}\left(t=t_{s}\right)$, the steady function can be obtained in a straightforward way.

\section{Preliminary Results}

We applied our method to p-type silicon at $300 \mathrm{~K}$, as a check. The valence band is supposed: non-degenerate, non-parabolic, and spherical. I and $J$ are fixed to 6 . In Figure 2, steady distribution function is represented from 0 to $20 \mathrm{kV} \mathrm{cm}^{-1}$, for a null doping level. On the other hand, Figure 3 shows the transient regime carrier density for $20 \mathrm{kV} \mathrm{cm}{ }^{-1}$. As forecasted, the distribution function spreads along the $k$-axis, when the electric field grows. Finally, transient drift velocity (Fig. 4) and mean energy (Fig. 5) are presented for 5,10 and $20 \mathrm{kV} \mathrm{cm}{ }^{-1}$, respectively. We remark the overshoot velocity enhanced with the electric field. We compared systematically our method to the Monte-Carlo one (a direct method). As it can be seen in these figures, the comparison appears quite good (Monte-Carlo results being fitted by using a least mean squares algorithm).

\section{Conclusion}

We present in this paper a new method to solve the Boltzmann equation. For that, we expand the distribution function in a Laguerre and Legendre polynomial set. It is less exact than direct technique as the Monte-Carlo one, since it uses a least-square fitting algorithm. In fact, comparison with the Monte-Carlo resolution leads to a very good agreement. 
The key point to be noted is its fast computing time: 200 faster than the Monte-Carlo method for the same accuracy. The basic interest of this new method (the fast computation) is due that all of the integrals are not dependent on time, and then, they can be calculated at the begimning of the programme.

The future trends will be to apply this algorithm at the nitrogen ambient, for P-Si and III-V compounds. Moreover, we think this polynomial expansion, coupled with Poisson's equation, should be a good tool to model submicron devices.

\section{References}

[1] Budd H.F., Proceeding of the International Conference on the Physics of semiconductors, (kyoto, 1966), J. Phys. Soc. Jpn. Suppl. 21 (1966) 420.

[2] Rees H.D., Phys. Lett. 26A (1968) 416.

[3] Kurosawa T., Proceeding of the International Conference on the Physics of semiconductors (kyoto, 1966) J. Phys. Soc. Jpn. Suppl. 21 (1966) 424.

[4] Hänsch W. and Miura-Mattausch M., The Hot-Electron Problem in Small Semiconductor Devices, J. Appl. Phys. 2 (1986) 650-656.

[5] Schöll E. and Quade W., Effect of Impact Ionisation on Hot-Carrier Energy and Momentum Relaxation in Serniconductor, J. Phys. C: Solid State Phys. 20 (1987) L861-L867.

[6] Wolff P.A., Theory of Multiplication in Silicon and Germanium, Phys. Rev. 95 (1954) $1415-1420$.

[7] Blotekjaer K., Transport equations for electrons in two-valley Semiconductors, IEEE Trans. Electron Dev. ED-17 (1970) 38-47.

[8] Rosencher E.A, Displaced Maxwellian Approach to Ballistic Electron Transport in Semiconductors, Solid State Communications 38 (1981) 1293-1295.

[9] van Someren Greve SC, Thesis, Technische hogeschool (Eindhoven, 1984).

[10] Nougier J.F., Tabikh S. and Vaissiere J.C., Fast Computation of transport coefficients of hot carriers, Semicond. Sci. Technol. 7 (1992) B351-B353. 
